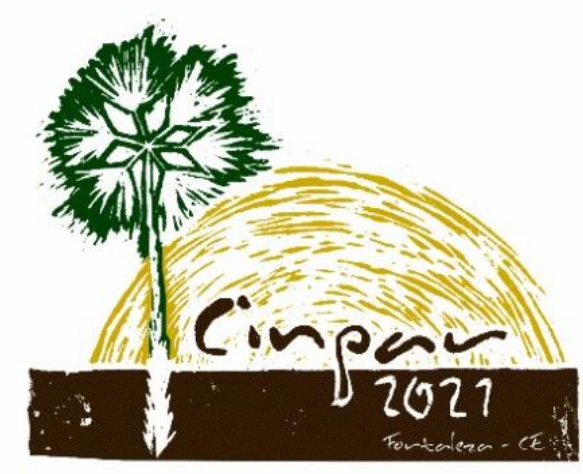

XVII Congresso Internacional sobre Patologia e Reabilitação das Construções

XVII Congreso Internacional sobre Patología y Rehabilitación de las Construcciones

XVII International Conference on Pathology and Constructions Rehabilitation

FORTALEZA (Brasil), 3 a 5 de junho de 2021 https://doi.org/10.4322/CINPAR.2021.060

\title{
Caracterização das propriedades físicas da espécie de bambu Bambusa Vulgaris cultivada em Redenção, Ceará-Brasil
}

\section{Characterization of the physical properties of the Bambusa Vulgaris bamboo species grown in Redenção, Ceará-Brazil}

\author{
Wanner Kelly DAMASCENO DA SILVA', Tallis Deyvide MAIA RUBENS², Antônio Eduardo BEZERRA CABRAL ${ }^{3}$ \\ ${ }^{1}$ Universidade Federal do Ceará UFC, Fortaleza, Brasil, kellydamasc@gmail.com1 \\ ${ }^{2}$ Universidade Federal do Ceará UFC, Fortaleza, Brasil, tallis.maia@gmail.com2 \\ ${ }^{3}$ Universidade Federal do Ceará UFC, Fortaleza, Brasil, eduardo.cabral@ufc.br3
}

\begin{abstract}
Resumo
A elevada produtividade, o crescimento rápido, o baixo custo e o fácil cultivo tornam o bambu um material sustentável promissor como alternativa em projetos de engenharia e arquitetura. No entanto, o escasso conhecimento acadêmico sobre a espécie Bambusa Vulgaris, a sua utilização incipiente na construção civil e a sua alta oferta na região Nordeste do Brasil evidenciam a necessidade de geração de conhecimento quanto às características do bambu. $O$ presente trabalho tem como objetivo analisar as características físicas do bambu de espécie Bambusa Vulgaris, extraído de três regiões distintas (basal, mediana e topo; com e sem nó) do colmo maduro cultivado no município de Redenção, Ceará, Brasil. Foram realizados ensaios de retração e inchamento para avaliação da estabilidade dimensional do bambu; também foram analisados o teor de umidade e as densidades básica e aparente, a fim de evidenciar o fácil manuseio do material. Os resultados de caracterização física da espécie de bambu B. Vulgaris evidenciam sua potencial aplicação na construção civil como material alternativo, tendo em vista os valores de retração e inchamento volumétricos na ordem média de $0,6 \%$ e $2,6 \%$, respectivamente, em $24 \mathrm{~h}$, comparativos à madeira Eucalipto com retração média de $0,9 \%$ e inchamento superior a $10 \%$.
\end{abstract}

Palavras-chave: Sustentabilidade; Construção civil; Caracterização; Material Alternativo; Bambu.

\begin{abstract}
High productivity, rapid growth, low cost and easy cultivation make bamboo a promising sustainable material as an alternative in engineering and architecture projects. However, the lack of academic knowledge about the species Bambusa Vulgaris, its incipient use in civil construction and its high supply in the Northeast region of Brazil show the need to generate knowledge about the characteristics of bamboo. The present work aims to analyze the physical characteristics of Bambusa Vulgaris bamboo, extracted from three distinct regions (basal, median and top; with and without a knot) of the mature stem grown in the municipality of Redenção, Ceará, Brazil. Retraction and swelling tests were carried out to assess the dimensional stability of the bamboo; the moisture content and the basic and apparent densities were also analyzed, in order to demonstrate the easy handling of the material. The results of physical characterization of the bamboo species B. Vulgaris show its potential application in civil construction as an alternative material, bearing in mind the volumetric shrinkage and swelling values in the average order of $0.6 \%$ and $2.6 \%$, respectively, in $24 \mathrm{~h}$, compared to Eucalyptus wood with an average retraction of $0.9 \%$ and swelling greater than $10 \%$.
\end{abstract}

Keywords: Sustainability; Construction; Characterization; Alternative material; Bamboo. 


\section{Introdução}

A China, líder na produção mundial de bambu, tem uma vasta lista de utilidades da planta, cerca de 1500 aplicações. Compõem essa lista o seu emprego em projetos de irrigação, paisagismo e artesanato, bem como o aproveitamento na culinária, na produção de papel, carvão, móveis e em projetos de engenharia e arquitetura (RIBAS, 2015). Na Índia, é utilizado como matéria-prima para celulose. O papel feito de Bambusa Vulgaris tem excepcional resistência ao rasgo, comparável ao papel feito de madeira macia. Também pode ser usado para fazer painéis de partículas e papel de grau de embalagem flexível.

No território brasileiro, o bambu é cultivado principalmente nas regiões nordeste e sudeste do país. No estado do Maranhão, a sua produção é destinada para a geração de energia através da biomassa. Nos estados de Pernambuco e Paraíba, o bambu é utilizado na produção de celulose e papel, já a produção de painéis, broto e fitocosméticos, se concentram nos estados de São Paulo, Minas Gerais, Bahia, Paraná e Rio Grande do Sul (EMBRAPA, 2018).

Por apresentar cultivo fácil e de baixo custo, crescimento rápido, elevada produtividade por hectare, baixa densidade, alta resistência à tração e compressão, e baixa energia de produção, o bambu e a sua madeira tornaram-se alternativas sustentáveis aos materiais convencionais que compõem os projetos de engenharia e arquitetura. Por essas e outras características, o bambu possui elevado potencial de aplicação na construção civil, em especial nos elementos que compõem as edificações, sejam eles estruturais, como: pilares, vigas e lajes, ou arquitetônicos, mesclando funções mecânicas à estética da planta. No entanto, quando utilizado em projetos estruturais, é fortemente recomendada a aplicação de tratamentos preventivos, pois como todo material biológico, o bambu está suscetível à deterioração natural (GHAVAMI E TOLEDO FILHO, 1992).

Em alguns países asiáticos, latino-americanos e africanos, os colmos de bambu são tradicionalmente usados como vigas e colunas (YU, W. K.; CHUNG, K. F.; CHAN, S. L., 2005; KAMINSKI, LAWRENCE e TRUJILLO, 2016). Além disso, os colmos de bambu também podem ser usados para realizar produtos de engenharia, como compósitos laminados e scrimber (VERMA e CHARIAR, 2012; SHARMA et al., 2015).

O bambu, como material alternativo em substituição parcial ou total aos convencionais da construção civil, como: o concreto, a madeira e o aço; pode contribuir para a redução da emissão do gás carbônico na atmosfera, para a diminuição do desmatamento de florestas virgens e para a redução da geração de resíduos sólidos (PADOVAN, 2010).

O alto desempenho mecânico aliado à sustentabilidade confere ao bambu um alto potencial para substituir materiais de construção convencionais em diversas aplicações. Em particular, existem países em que este material é utilizado na construção há milênios e representa uma mais-valia, pelo contrário, existem países onde ainda não existe conhecimento suficiente das propriedades estruturais do bambu cultivado localmente. Nestes casos, é importante ampliar o conhecimento das propriedades das espécies locais de bambu, apoiado no desenvolvimento de procedimentos de teste padronizados adequados (MOLARI, MENTRASTI e FABIANI, 2020).

O presente estudo tem por objetivo caracterizar o bambu de espécie Bambusa Vulgaris quanto às propriedades físicas de densidades básica e aparente, teor de umidade, retração e inchamento volumétricos. Foram investigadas as diferenças entre os valores das propriedades mencionadas em diferentes posições do colmo (Base, Intermédio e Topo) e na presença ou ausência de nós, visando indicar a utilização do bambu $B$. Vulgaris como material alternativo para construção civil.

\section{Materiais e Métodos}

\subsection{Bambu da espécie Bambusa Vulgaris}

Foi selecionado para o presente estudo o bambu de espécie Bambusa Vulgaris, predominante do estado do Ceará/Brasil. Todos os colmos de bambu testados foram cultivados em Redenção, município localizado a 70 $\mathrm{km}$ da capital do estado cearense, cujo clima é Tropical Quente Úmido, caracterizado por altas temperaturas e elevada umidade, favorável ao desenvolvimento desta espécie de bambu. A espécie 
estudada faz parte da categoria de bambu simpodial ou entouceirante (Figura 1), cujos rizomas são curtos e crescem próximos uns dos outros, formando grupos de bambus em touceiras aglomeradas.

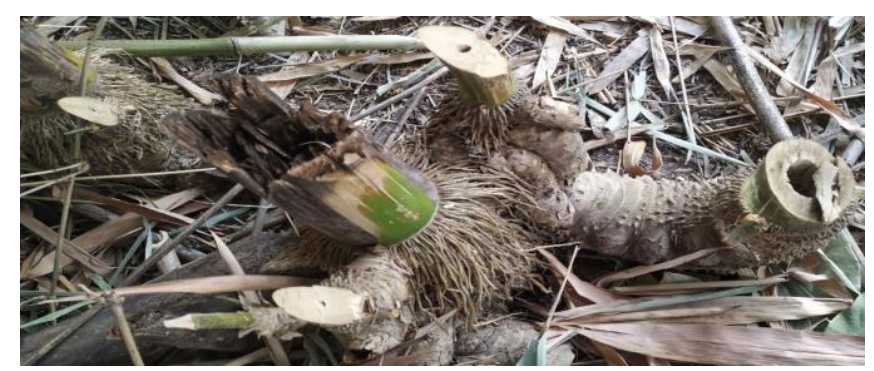

Figura 1 - Rizoma do tipo Simpodial ou Entouceirante

A seleção dos colmos de bambu foi realizada de forma aleatória, no entanto buscando-se amostras representativas com idade média entre três e cinco anos de plantação, tendo em vista que varas mais maduras e livres de defeitos aparentes apresentam maior rigidez dos colmos. Para identificação das varas maduras, observou-se a ausência de brácteas; a presença de manchas externas; o aspecto envelhecido e a posição da touceira, normalmente ao centro do bambuzal e com acesso dificultado.

Após coleta, as varas foram transportadas e abrigadas em local livre de intempéries, como chuva e incidência solar, e posicionadas na vertical para secagem natural ao ar.

As varas de bambu foram cortadas no equipamento Serra de bancada (Corrupio) com disco de corte apropriado para madeira (Figura 2 a) nas dimensões especificadas em cada ensaio (Figura 2 b). Os colmos foram divididos em três posições distinta, sendo elas identificadas como Base (B), Intermédio (I) e Topo (T). Ainda, as amostras contemplam a variável Com ou Sem nó, portanto, foram distribuídas em seis grupos de análise (Basal sem nó, Intermédio sem nó, Topo sem nó, Basal com nó, Intermédio com nó e Topo com nó).

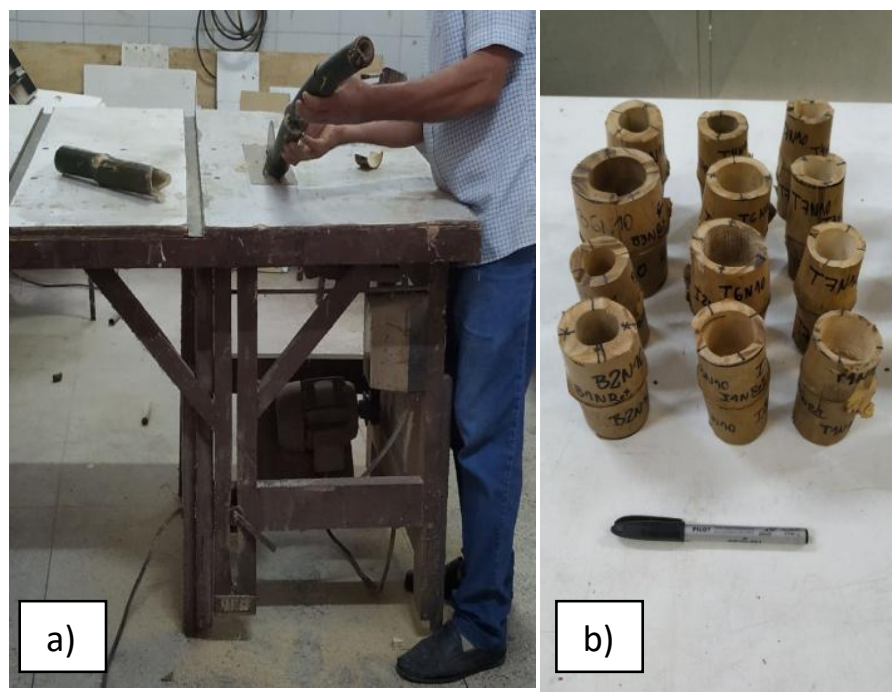

Figura 2 - a) Corte do colmo para confecção dos corpos de prova. b) Amostras de corpos de prova em bambu

2.2 Análise das propriedades físicas do bambu

Foram realizados os ensaios de caracterização física do bambu da espécie Bambusa Vulgaris conforme descrito no Quadro 1. 
Quadro 1 - Caracterização física do Bambusa Vulgaris

\begin{tabular}{|c|c|c|c|c|}
\hline Determinação & Método & Equipamento & Amostragem & Dimensões (mm) \\
\hline Teor de umidade & $\begin{array}{c}\text { ISO 22157:2019/ } \\
\text { NBR 16828-2: 2019 }\end{array}$ & $\begin{array}{c}\text { Balança / } \\
\text { Estufa }\end{array}$ & 24 unidades & $25 \times 25 \times \mathrm{t}^{*}$ \\
\hline $\begin{array}{c}\text { Densidade } \\
\text { básica }\end{array}$ & $\begin{array}{c}\text { ISO 22157:2019/ } \\
\text { NBR 16828-2: } 2019\end{array}$ & $\begin{array}{c}\text { Paquímetro / } \\
\text { Balança / } \\
\text { Estufa }\end{array}$ & 24 unidades & $25 \times 25 \times \mathrm{t}^{*}$ \\
\hline $\begin{array}{c}\text { Densidade } \\
\text { Aparente }\end{array}$ & NBR 7190:1997 & $\begin{array}{c}\text { Paquímetro / } \\
\text { Balança }\end{array}$ & 24 unidades & $25 \times 25 \times \mathrm{t}^{*}$ \\
\hline Retração & NBR 16828-2: 2019 & $\begin{array}{c}\text { Paquímetro / } \\
\text { Estufa }\end{array}$ & 24 unidades & $100 \times \mathrm{D} \times \mathrm{t}^{*}$ \\
\hline Inchamento & $\begin{array}{r}\text { NBR 16828-2: } 2019 \\
\text { Adaptado }\end{array}$ & Paquímetro & 24 unidades & $100 \times \mathrm{D} \times \mathrm{t}^{*}$ \\
\hline
\end{tabular}

Nota: D: diâmetro externo; t: espessura da parede do colmo

\section{Apresentação e discussão dos resultados}

Os valores médios de densidade básica do Bambusa Vulgaris são apresentados na Tabela 1, em conjunto aos desvios e ao coeficiente de variação das médias. Observa-se maior densidade básica média para amostras com nós em relação às três regiões do bambu (Base, Intermédio e Topo), este resultado é justificado pela concentração de fibras na região nodal. Verifica-se o crescimento da densidade básica média no sentido da base ao topo do colmo, Brito et al (2015) explicam este incremento devido à grande concentração dos feixes fibrovasculares na região do topo, somado à redução das células parenquimáticas nessa posição para espécies de bambu Dendrocalamus giganteus Munro. Comportamento similar é relatado nos estudos de Azzini et al (1987), com valores variando de $660 \mathrm{~kg} / \mathrm{m}^{3}$ a $813 \mathrm{~kg} / \mathrm{m}^{3}$; Lima et al (2011), com resultados de $400 \mathrm{~kg} / \mathrm{m}^{3}$ a $550 \mathrm{~kg} / \mathrm{m}^{3}$; Moreira (2012), apresentou valores entre $538 \mathrm{~kg} / \mathrm{m}^{3}$ a $591 \mathrm{~kg} / \mathrm{m}^{3}$; Balduíno Júnior (2015), relatou valores entre $540 \mathrm{~kg} / \mathrm{m}^{3}$ e $689 \mathrm{~kg} / \mathrm{m}^{3}$, e Brand et al (2020), valor médio de densidade básica igual a $623 \mathrm{~kg} / \mathrm{m}^{3}$.

Tabela 1 - Densidade básica do bambu Bambusa Vulgaris

\begin{tabular}{|c|c|c|c|c|c|}
\hline \multicolumn{2}{|c|}{$\begin{array}{c}\text { Posição } \\
\text { no colmo }\end{array}$} & $\begin{array}{c}\text { Densidade } \\
\text { Básica média } \\
\left(\mathbf{k g} / \mathbf{m}^{3}\right)\end{array}$ & $\begin{array}{c}\text { Desvio } \\
\text { Padrão } \\
\left(\mathbf{k g} / \mathbf{m}^{3}\right)\end{array}$ & $\begin{array}{c}\text { Desvio } \\
\text { Médio } \\
\left(\mathbf{k g} / \mathbf{m}^{\mathbf{3}}\right)\end{array}$ & $\begin{array}{c}\text { CV } \\
(\%)\end{array}$ \\
\hline \multirow{2}{*}{ Base } & Com nó & 462 & 126 & 97 & 27 \\
\cline { 2 - 6 } & Sem nó & 347 & 60 & 50 & 17 \\
\hline \multirow{3}{*}{ Intermédio } & Com nó & 489 & 68 & 54 & 14 \\
\cline { 2 - 6 } & Sem nó & 480 & 17 & 13 & 4 \\
\hline \multirow{2}{*}{ Topo } & Com nó & 495 & 35 & 29 & 7 \\
\cline { 2 - 6 } & Sem nó & 471 & 46 & 37 & 10 \\
\hline \multicolumn{2}{|c|}{ Valores médios } & 457 & 59 & 47 & 13 \\
\hline
\end{tabular}

Nota: CV: Coeficiente de Variação

Foi obtido valor médio igual a $457 \mathrm{~kg} / \mathrm{m}^{3}$, similar ao resultado observado por Lima et al (2011), onde o valor máximo de densidade básica média para o presente estudo foi de $495 \mathrm{~kg} / \mathrm{m}^{3}$ na região do topo com nó e valor mínimo de $347 \mathrm{~kg} / \mathrm{m}^{3}$ para a posição da base sem nó. Em comparação a espécie de madeira Eucalyptus sp., observam-se valores próximos ao obtido para o Bambusa Vulgaris, onde Protásio et al. 
(2013) evidenciam valores de densidade entre $471 \mathrm{~kg} / \mathrm{m}^{3}$ a $619 \mathrm{~kg} / \mathrm{m}^{3}$ para diferentes espécies de Eucalyptus, indicando o potencial do uso do bambu na construção civil.

A Tabela 2 apresenta valores médios para a densidade aparente do Bambusa Vulgaris, onde observa-se comportamento similar ao constatado para a densidade básica média, sendo os maiores valores obtidos para amostras com a presença de nós e o crescimento desta propriedade física no sentido base-topo. Comparando-se a densidade básica média à densidade aparente média, observa-se que esta é $22 \%$ menor que a densidade básica, visto que a última considera os vazios existentes na amostra.

Foi obtido valor médio para a densidade aparente igual a $356 \mathrm{~kg} / \mathrm{m}^{3}$, onde o valor máximo foi registrado na região do intermédio com nó igual a $387 \mathrm{~kg} / \mathrm{m}^{3}$ e valor mínimo de $281 \mathrm{~kg} / \mathrm{m}^{3}$ para a posição basal sem nó. Resultados similares foram constatados por Balduíno Júnior (2015), o qual relatou valores entre $323 \mathrm{~kg} / \mathrm{m}^{3}$ e $431 \mathrm{~kg} / \mathrm{m}^{3}$ para carvão vegetal de Bambusa Vulgaris; Brand et al (2020), valores para densidade aparente entre 323 a $431 \mathrm{~kg} / \mathrm{m}^{3}$; ambos os estudos evidenciando o incremento de densidade da base ao topo.

Tabela 2 - Densidade aparente do bambu Bambusa Vulgaris

\begin{tabular}{|c|c|c|c|c|c|}
\hline \multicolumn{2}{|c|}{$\begin{array}{c}\text { Posição } \\
\text { no colmo }\end{array}$} & $\begin{array}{c}\text { Densidade } \\
\text { Aparente média } \\
\left(\mathbf{k g} / \mathbf{m}^{3}\right)\end{array}$ & $\begin{array}{c}\text { Desvio } \\
\text { Padrão } \\
\left(\mathbf{k g} / \mathbf{m}^{3}\right)\end{array}$ & $\begin{array}{c}\text { Desvio } \\
\text { Médio } \\
\left(\mathbf{k g} / \mathbf{m}^{3}\right)\end{array}$ & $\begin{array}{c}\text { CV } \\
\mathbf{( \% )}\end{array}$ \\
\hline \multirow{2}{*}{ Base } & Com nó & 371 & 80 & 61 & 22 \\
\cline { 2 - 6 } & Sem nó & 281 & 41 & 27 & 15 \\
\hline \multirow{3}{*}{ Intermédio } & Com nó & 387 & 57 & 48 & 15 \\
\cline { 2 - 6 } & Sem nó & 349 & 16 & 11 & 4 \\
\hline \multirow{2}{*}{ Topo } & Com nó & 378 & 22 & 16 & 6 \\
\cline { 2 - 6 } & Sem nó & 369 & 23 & 16 & 6 \\
\hline \multicolumn{2}{|c|}{ Valores médios } & 356 & 40 & 30 & 11 \\
\hline
\end{tabular}

Nota: CV: Coeficiente de Variação

O teor de umidade natural da espécie Bambusa Vulgaris foi verificado após 52 dias, 100 dias e 101 dias da coleta dos colmos. Os resultados são apresentados na Figura 3, para amostras sem nó, e Figura 4, para corpos de prova com nó. Observa-se a diminuição acentuada do teor de umidade ao longo do tempo para as três diferentes posições do bambu, constatando-se maior variação para a região do topo com e sem nó.

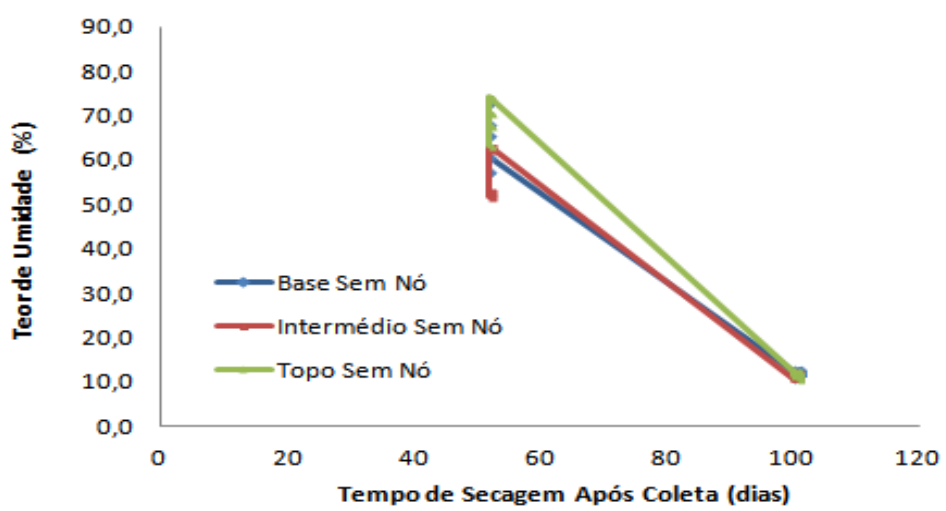

Figura 3 - Teor de umidade em função do tempo de secagem do bambu Bambusa Vulgaris após coleta (Amostras Sem Nó) 


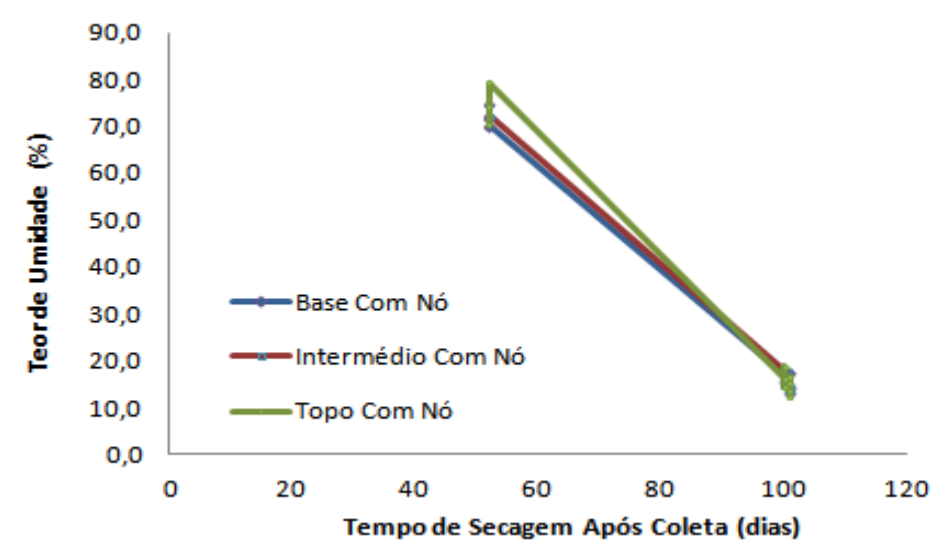

Figura 4 - Teor de umidade em função do tempo de secagem do bambu Bambusa Vulgaris após coleta (Amostras Com Nó)

Maiores valores de umidade foram obtidos para amostras na região do topo após 52 dias e 100 dias da coleta dos colmos, conforme Tabela 3, no entanto ao terceiro dia foi constatada a redução da umidade no sentido longitudinal, ou seja, da base ao topo. Tal resultado pode ser explicado pela redução dos lumes e pelo aumento da espessura das paredes dos feixes fibrovasculares, constatado nos estudos de Berndsen (2008) e Brito et al. (2015).

Tabela 3 - Teor de umidade média do bambu Bambusa Vulgaris

\begin{tabular}{|c|c|c|c|c|c|c|c|}
\hline \multirow{2}{*}{$\begin{array}{c}\text { Posição } \\
\text { no colmo }\end{array}$} & \multicolumn{2}{|c|}{ Teor de Umidade média (\%) } & \multicolumn{3}{c|}{ Desvio Padrão (\%) } \\
\cline { 2 - 8 } & $\mathbf{5 2}$ dias & $\mathbf{1 0 0}$ dias & $\mathbf{1 0 1}$ dias & $\mathbf{5 2}$ dias & $\mathbf{1 0 0}$ dias & $\mathbf{1 0 1}$ dias \\
\hline \multirow{2}{*}{ Base } & Com nó & 72,1 & 16,2 & 15,1 & 1,9 & 1,0 & 1,9 \\
\cline { 2 - 8 } & Sem nó & 64,9 & 12,1 & 12,1 & 6,0 & 0,7 & 0,3 \\
\hline \multirow{3}{*}{ Intermédio } & Com nó & 72,2 & 16,0 & 14,3 & 0,1 & 1,3 & 0,4 \\
\cline { 2 - 8 } & Sem nó & 57,6 & 11,7 & 11,7 & 5,9 & 0,7 & 0,5 \\
\hline \multirow{2}{*}{ Topo } & Com nó & 75,1 & 16,5 & 14,5 & 3,6 & 1,9 & 1,9 \\
\cline { 2 - 8 } & Sem nó & 68,9 & 12,4 & 11,5 & 4,6 & 0,5 & 0,7 \\
\hline
\end{tabular}

Nota: CV: Coeficiente de Variação

Foi analisada a estabilidade dimensional do Bambusa Vulgaris através da retração e do inchamento volumétricos, a fim de determinar a utilização desta espécie de bambu na construção civil. A Figura 5 apresenta valores de retração volumétrica ao longo do tempo (24h, 48h, 72h e 96h) para amostras sem nó, na qual se constatam maiores valores médios de retração para a base, atingindo a retração máxima de 1,6\% após $96 \mathrm{~h}$ de secagem em estufa, também evidenciado na Tabela 4. 


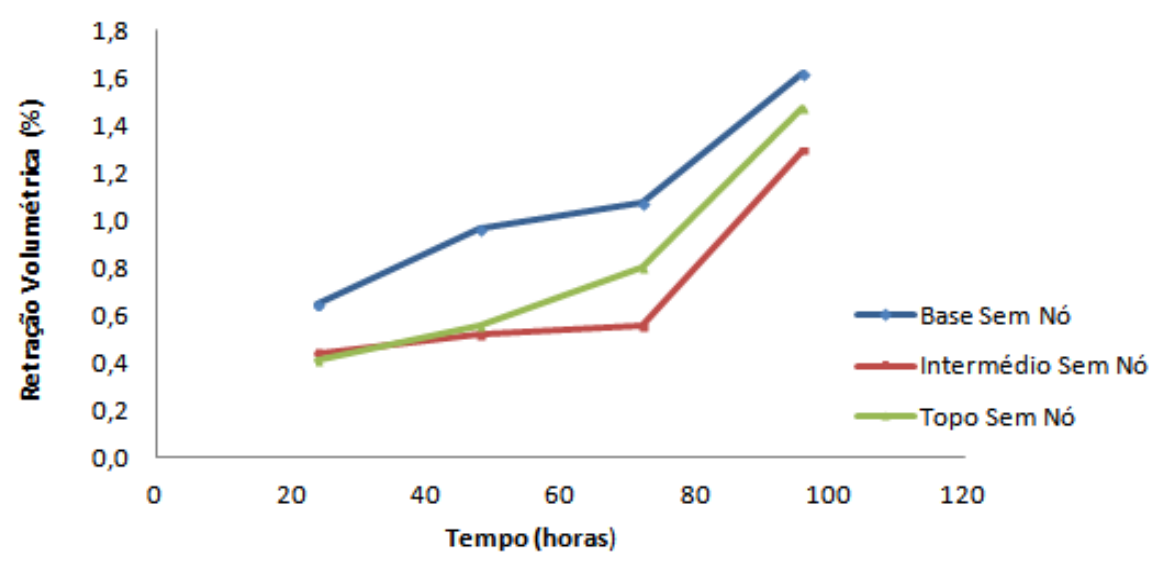

Figura 5 - Retração volumétrica em função do tempo (Amostras Sem Nó)

A Figura 6 apresenta valores de retração volumétrica em relação ao tempo para amostras com nó, onde se constatam maiores valores médios de retração para a região intermediária, atingindo a retração máxima de $1,5 \%$ após $96 \mathrm{~h}$ de secagem em estufa, conforme Tabela 4.

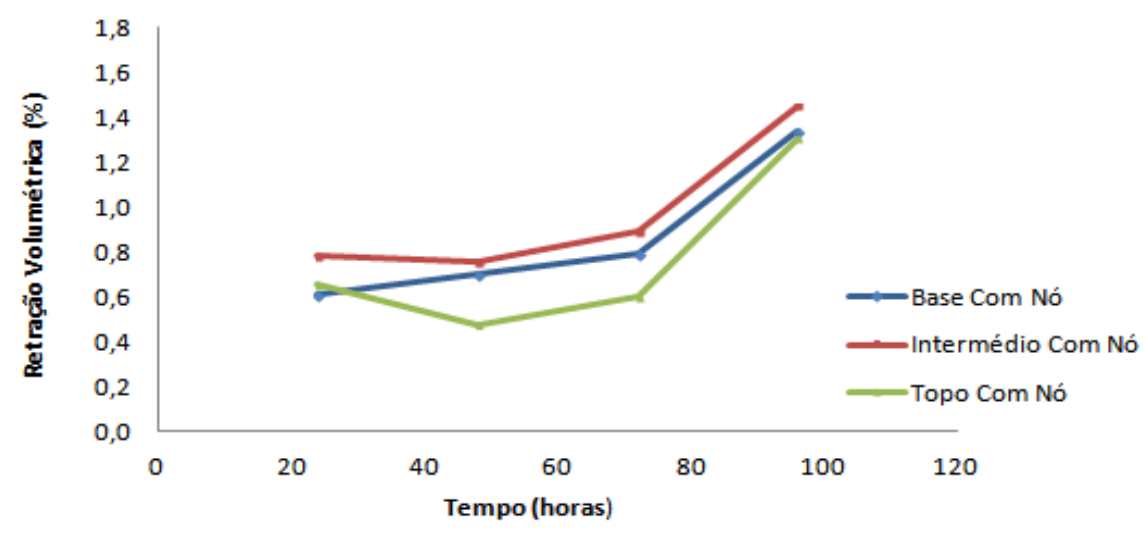

Figura 6 - Retração volumétrica em função do tempo (Amostras Com Nó)

Constataram-se valores discrepantes para a retração volumétrica existente no topo e no intermédio após $24 \mathrm{~h}$ de ensaio, onde foram obtidos valores superiores à idade de $48 \mathrm{~h}$. Não foram observadas discrepâncias similares nas demais idades.

Tabela 4 - Retração volumétrica média do bambu Bambusa Vulgaris

\begin{tabular}{|c|c|c|c|c|c|}
\hline \multirow{2}{*}{$\begin{array}{c}\text { Posição } \\
\text { no colmo }\end{array}$} & \multicolumn{4}{|c|}{ Inchamento Volumétrico médio (\%) } \\
\cline { 2 - 6 } & $\mathbf{2 4}$ horas & $\mathbf{4 8}$ horas & $\mathbf{7 2}$ horas & $\mathbf{9 6}$ horas \\
\hline \multirow{2}{*}{ Base } & Com nó & 0,6 & 0,7 & 0,8 & 1,3 \\
\cline { 2 - 6 } & Sem nó & 0,7 & 1,0 & 1,1 & 1,6 \\
\hline \multirow{3}{*}{ Intermédio } & Com nó & 0,8 & 0,8 & 0,9 & 1,5 \\
\cline { 2 - 6 } & Sem nó & 0,4 & 0,5 & 0,6 & 1,3 \\
\hline \multirow{2}{*}{ Topo } & Com nó & 0,7 & 0,5 & 0,6 & 1,3 \\
\cline { 2 - 6 } & Sem nó & 0,4 & 0,6 & 0,8 & 1,5 \\
\hline
\end{tabular}




\begin{tabular}{|c|c|c|c|c|}
\hline Valores médios & 0,6 & 0,7 & 0,8 & 1,4 \\
\hline
\end{tabular}

Observa-se, para as amostras com e sem nó, que não houve constância nos valores de retração volumétrica após $96 \mathrm{~h}$, sendo indicada a continuação desta análise para maiores idades.

A Figura 7 apresenta valores de inchamento volumétrico ao longo do tempo (24h, $48 \mathrm{~h}, 72 \mathrm{~h}$ e $96 \mathrm{~h}$ ) para amostras sem nó, na qual se constatam maiores valores médios de inchamento para o topo, atingindo valor máximo de $16,7 \%$ após $96 \mathrm{~h}$ de saturação em água destilada, também evidenciado na Tabela 5 . Observa-se um crescimento elevado no inchamento das amostras sem nó no intervalo entre $72 \mathrm{~h}$ e $96 \mathrm{~h}$, bem como não foi verificada a constância nos valores de inchamento após $96 \mathrm{~h}$, sendo indicada a continuação da análise dessa propriedade ao longo de maior período.

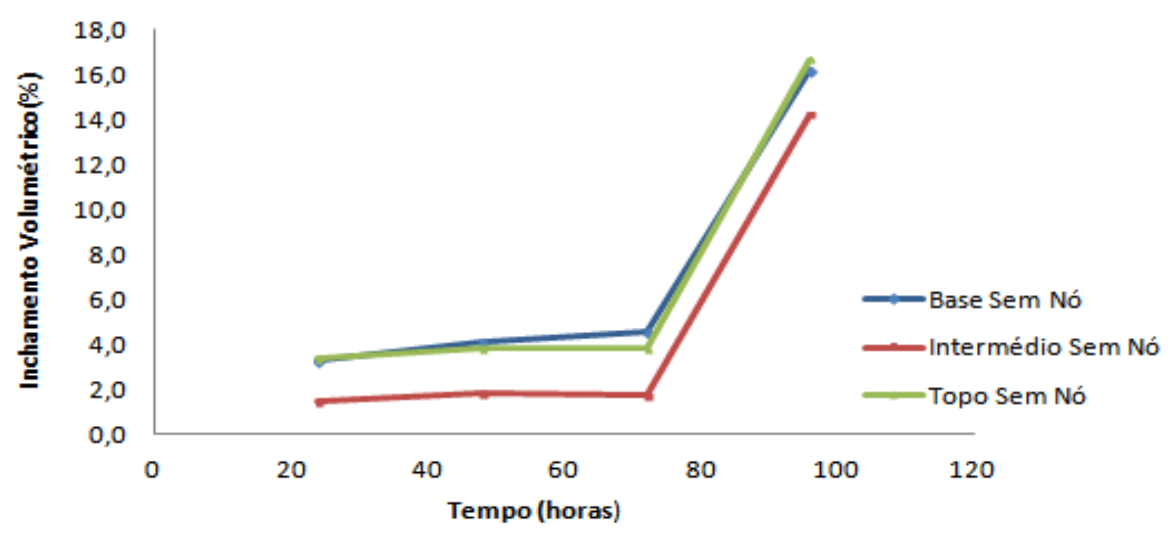

Figura 7 - Inchamento volumétrico em função do tempo (Amostras Sem Nó)

A Figura 8 apresenta valores de inchamento volumétrico em relação ao tempo para amostras com nó, onde se constatam maiores valores médios de inchamento para a região intermediária, atingindo valor máximo de 15,8\% após $96 \mathrm{~h}$ de saturação em água destilada, conforme Tabela 5.

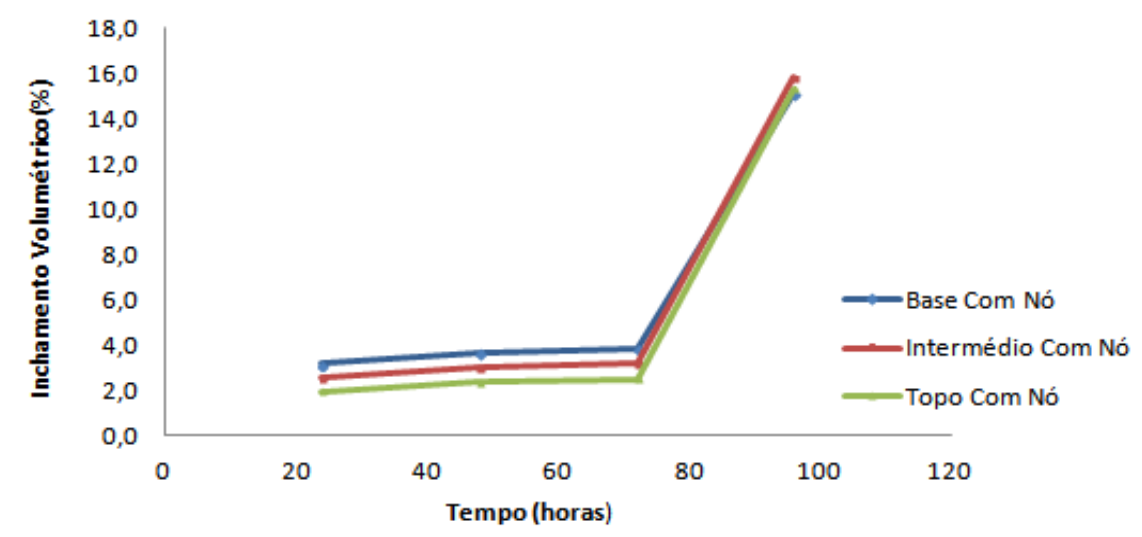

Figura 8 - Inchamento volumétrico em função do tempo (Amostras Com Nó)

Observam-se maiores valores de inchamento no sentido Base-Topo até a idade de $72 \mathrm{~h}$, havendo alteração às $96 \mathrm{~h}$ quando a região intermediária assume o maior valor de inchamento $(15,8 \%)$, posterior ao topo $(15,3 \%)$ e à região basal $(15,1 \%)$. 
Tabela 5 - Inchamento volumétrico médio do bambu Bambusa Vulgaris

\begin{tabular}{|c|c|c|c|c|c|}
\hline \multirow{2}{*}{\multicolumn{2}{|c|}{$\begin{array}{l}\text { Posição } \\
\text { no colmo }\end{array}$}} & \multicolumn{4}{|c|}{ Inchamento Volumétrico médio (\%) } \\
\hline & & \multirow{2}{*}{$\frac{24 \text { horas }}{3,2}$} & \multirow{2}{*}{$\frac{48 \text { horas }}{3,7}$} & \multirow{2}{*}{$\frac{72 \text { horas }}{3,9}$} & \multirow{2}{*}{$\frac{96 \text { horas }}{15,1}$} \\
\hline & Com nó & & & & \\
\hline (5) & Sem nó & 3,3 & 4,1 & 4,6 & 16,2 \\
\hline \multirow{2}{*}{ Intermédio } & Com nó & 2,6 & 3,1 & 3,2 & 15,8 \\
\hline & Sem nó & 1,5 & 1,8 & 1,8 & 14,2 \\
\hline \multirow{2}{*}{ Topo } & Com nó & 2,0 & 2,4 & 2,5 & 15,3 \\
\hline & Sem nó & 3,4 & 3,9 & 3,9 & 16,7 \\
\hline \multicolumn{2}{|c|}{ Valores médios } & 2,6 & 3,2 & 3,3 & 15,5 \\
\hline
\end{tabular}

\section{Conclusões}

Foi realizada a caracterização física do bambu de espécie Bambusa Vulgaris, sendo realizada uma análise comparativa entre os valores de densidades básica e aparente, teor de umidade, retração e inchamento volumétricos em diferentes posições do colmo (Base, Intermédio e Topo), na presença ou ausência de nós, visando indicar a utilização do bambu $B$. Vulgaris como material alternativo à construção civil.

Com a realização da caracterização física das amostras, observou-se uma maior densidade básica média para amostras com nós em relação às três regiões do bambu (Base $\left(462 \mathrm{~kg} / \mathrm{m}^{3}\right)$, Intermédio $\left(489 \mathrm{~kg} / \mathrm{m}^{3}\right)$ e Topo $\left.\left(495 \mathrm{~kg} / \mathrm{m}^{3}\right)\right)$, este resultado é justificado pela concentração de fibras na região nodal. Foi verificado que a densidade básica média cresce no sentido da base ao topo do colmo, característica explicada pela grande concentração dos feixes fibrovasculares na região do topo. Foi obtido valor médio para a densidade básica média igual a $457 \mathrm{~kg} / \mathrm{m}^{3}$.

O teor de umidade natural da espécie Bambusa Vulgaris foi verificado após 52 dias, 100 dias e 101 dias da coleta dos colmos. Observou-se a diminuição acentuada do teor de umidade ao longo do tempo para as três diferentes posições do bambu, constatando-se maior variação para a região do topo com e sem nó.

Os resultados de caracterização física da espécie de bambu $B$. Vulgaris evidenciam sua potencial aplicação na construção civil como material alternativo, tendo em vista os valores de retração e inchamento volumétricos na ordem média de $0,6 \%$ e $2,6 \%$, respectivamente, em $24 \mathrm{~h}$, comparativos à madeira Eucalipto com retração média de $0,9 \%$ e inchamento superior a $10 \%$. Portanto, o caráter de contribuição deste trabalho se evidencia pela ampliação do conhecimento quanto às propriedades físicas do bambu de espécie B. Vulgaris, incentivando o uso de materiais eco-sustentáveis na construção civil brasileira, assim, empregando matérias-primas que permitam a redução de resíduos e poluição.

\section{Referências Bibliográficas}

Azzini, A.; Arruda, M. C.; Tomazzelo Filho, M.; Salgado, A. L., \& Ciaramello, D. (1987).Variações dos teores de fibras celulósicas e amido no colmo de bambu. Bragantia, Vol. 46(1), 141-145.

Balduino Junior, A. L. (2015). Avaliação do potencial energético da biomassa de duas espécies de bambu cultivadas em Santa Catarina. Diss. Universidade do Estado de Santa Catarina.

Berndsen, R. S. (2008). Caracterização anatômica, física e mecânica de lâminas de bambu (Phyllostachys pubescens). Diss. Dissertação de Mestrado em Engenharia Mecânica e de Materiais, Área de Concentração em Engenharia de Materiais) - Universidade Tecnológica Federal do Paraná, Curitiba, PR.

Brand, M. A., GAA, A. Z. N., Balduino Junior, A. L., Cunha, A. B. D., \& Rios, P. D. A. (2020). Potencial do uso de quatro espécies de bambu para a produção de carvão vegetal para uso doméstico. Ciência Florestal, Vol. 30(1), 60-71. 
Brito, F. M. S., Paes, J. B., Oliveira, J. T. D. S., Arantes, M. D. C., \& Fantuzzi Neto, H. (2015). Caracterização anatômica e física do bambu gigante (Dendrocalamus giganteus Munro). Floresta e Ambiente, Vol. 22.4: 559-566.

Embrapa (2018). Bambu é alternativa de renda na produção familiar. Disponível em: https://www.embrapa.br/busca-de-noticias/-/noticia/34230725/bambu-e-alternativa-de-renda-naproducao-familiar. Acesso em: 19 nov. de 2020.

Ghavami, K.; Toledo Filho, R. D. (1992). Desenvolvimento de Materiais de Construção de Baixo Consumo de Energia usando Fibras Naturais, Terra e Bambu. Revista Brasileira de Engenharia Agrícola, Publicação Sociedade Brasileira de Engenharia Agrícola. Vol.2 (1), p. 1-19.

KAMINSKI, Sebastian; LAWRENCE, Andrew; TRUJILLO, David. Structural use of bamboo: Part 1: Introduction to bamboo. The structural engineer, v. 94, n. 8, p. 40-43, 2016.

Molari, L.; Mentrasti, L.; Fabiani, M. Mechanical characterization of five species of Italian bamboo. In: Structures. Elsevier, 2020. p. 59-72.

Moreira, A. C. O. (2012). Caracterização de Bambusa vulgaris schard. ex j.c. wendl. var. vulgaris, e dos resíduos de caldeira no processo de conversão térmica de energia, dissertação de mestrado Universidade de Brasília - DF.

Padovan, R. B. (2010). O bambu na arquitetura: design de conexões estruturais. Diss. Dissertação de Mestrado. Programa de Pós-graduação em Design da Faculdade de Arquitetura, Artes e Comunicação. Universidade Estadual Paulista Júlio de Mesquita Filho, Bauru, SP.

Ribas, R. P. (2015). Bambu: Planta de Grande Potencial no Desenvolvimento Sustentável. Disponível em: https://mac.arq.br/wp-content/uploads/2016/03/bambu-desenvolvimento-sustentavel.pdf Acesso em: 20 nov. de 2020.

Sharma, B., Gatóo, A., Bock, M., \& Ramage, M. (2015). Engineered bamboo for structural applications. Construction and building materials, v. 81, p. 66-73.

Verma, C. S.; Chariar, V. M. (2012). Development of layered laminate bamboo composite and their mechanical properties. Composites Part B: Engineering, v. 43, n. 3, p. 1063-1069.

Yu, W. K.; Chung, K. F.; Chan, S. L. (2005). Axial buckling of bamboo columns in bamboo scaffolds. Engineering Structures, v. 27, n. 1, p. 61-73. 